\title{
Innovations sociales en Outaouais. Entre pratiques novatrices et innovations sociales
}

\author{
Denis Bourque, professeur \\ Lucie Fréchette, professeure \\ Jean Proulx, professionnel de recherche \\ Université du Québec en Outaouais
}

\section{INTRODUCTION}

Au cours des 20 dernières années, la région de l'Outaouais a vu naître des initiatives sociales qui ont été reconnues de succès. Certaines ont même franchi les frontières de la région pour se diffuser à travers le Québec. Le cas du Carrefour Jeunesse Emploi de l'Outaouais, expérience fondatrice des carrefours jeunesse emploi du Québec maintenant au nombre de 107 et le cas de La Relance Outaouais, première entreprise d'insertion à voir le jour au Québec, témoignent de l'inventivité outaouaise.
Constatant qu'un bon nombre d'initiatives de nature sociale prenaient la teneur de réussites, l'Agence de la santé et des services sociaux de l'Outaouais a commandité une recherche afin de documenter quelques réussites de la région sous l'angle de l'innovation sociale ${ }^{1}$. Les initiatives à succès de l'Outaouais constituaient-elles des innovations sociales? Quels sont les facteurs qui en ont influencé l'émergence, la consolidation et la diffusion? Ces questions ont retenu l'attention et sont traitées dans cet article.

\section{UNE ÉTUDE DU POINT DE VUE DE L'INNOVATION SOCIALE}

Documenter des pratiques sociales du point de vue de l'innovation exige d'abord un exercice de précision des concepts. Le concept d'innovation et, dans le domaine des sciences humaines et sociales, celui d'innovation sociale, ont gagné en popularité aux cours des dernières années. Les documents et les politiques du Québec et du Canada en matière de recherche et d'innovation qui voient le jour depuis le début des années 2000 en témoignent tout comme les travaux de grands organismes subventionnaires de recherche. Les débats et les recherches sur ces questions ont fait émerger quelques définitions, certaines s'appliquant plus à l'innovation, que l'on qualifie généralement de technologique, et d'autres référant plus à l'innovation associée à des programmes en réponse à des besoins sociaux. L'innovation technologique se situe d'abord dans une logique de marché. L'innovation sociale se situe plutôt dans le cadre du renouvellement de pratiques sociales. Elle produit des réponses inédites à des problèmes sociaux et ouvre de nouvelles avenues de déve- loppement des communautés locales. C'est dans ce large cadre de l'innovation qualifiée de sociale que se situe notre étude sur des pratiques innovantes en Outaouais.

\section{L'innovation sociale se situe plutôt dans le cadre du renouvellement de pratiques sociales. Elle produit des réponses inédites à des problèmes sociaux et ouvre de nouvelles avenues de développement des communautés locales.}

On peut dire sans nuancer que les travaux sur l'innovation ont été sujet d'intérêt d'abord en sciences économiques puis en sociologie ${ }^{2}$. L'innovation dans sa composante sociale a peu été théorisée sinon dans le champ socioéconomique sous l'angle plus spécifique de l'économie sociale ${ }^{3}$. Le développement social est cependant plus large que l'économie sociale et l'innovation y a souvent pour objectif la résolution de problèmes sociaux et socioéconomiques. L'innovation sociale qui accompagne le 
développement social n'émerge pas nécessairement d'un contexte d'urgence sociale ou de crise. Elle peut tout simplement résulter de l'aspiration à l'amélioration de la qualité de vie. Pour reprendre Castel et Parades, on dira de l'innovation sociale qu'elle est à la fois le résultat d'une histoire locale et le projet que se donne une organisation pour vivre sur un territoire ${ }^{4}$.

Dans cette recherche, nous avons retenu la définition de Camil Bouchard élaborée dans le cadre de travaux du Conseil québécois de la recherche sociale ${ }^{5}$ qui stipule que l'innovation sociale renvoie à «toute nouvelle approche, pratique, ou intervention ou encore tout nouveau produit pour améliorer une situation ou solutionner un problème social et ayant trouvé preneur au niveau des institutions, des organisations, des communautés».
La revue de littérature effectuée a amené à identifier différentes composantes de l'innovation sociale. Nous avançons l'idée que l'innovation sociale ne se limite pas à un produit achevé mais s'inscrit dans un processus. Le Réseau québécois en innovation sociale (RQIS) en est venu récemment à la même conclusion ${ }^{6}$. L'idée créative ou l'invention ont valeur en soi en tant que nouvelles façons de faire. Pour que ces inventions ou idées novatrices entraînent des changements sociaux, elles doivent cependant franchir la sphère d'influence de l'individu créatif ou celle du milieu qui a, en première instance, mis à l'essai l'invention. En s'appuyant sur les travaux issus de la littérature récente, un modèle a été conçu pour analyser les pratiques novatrices en Outaouais. Sans prétendre que toutes les innovations suivent le même parcours, ce modèle, illustré à la figure 1 , identifie les composantes jalonnant le processus d'innovation sociale.

Figure 1 : Composantes du processus d'innovation sociale

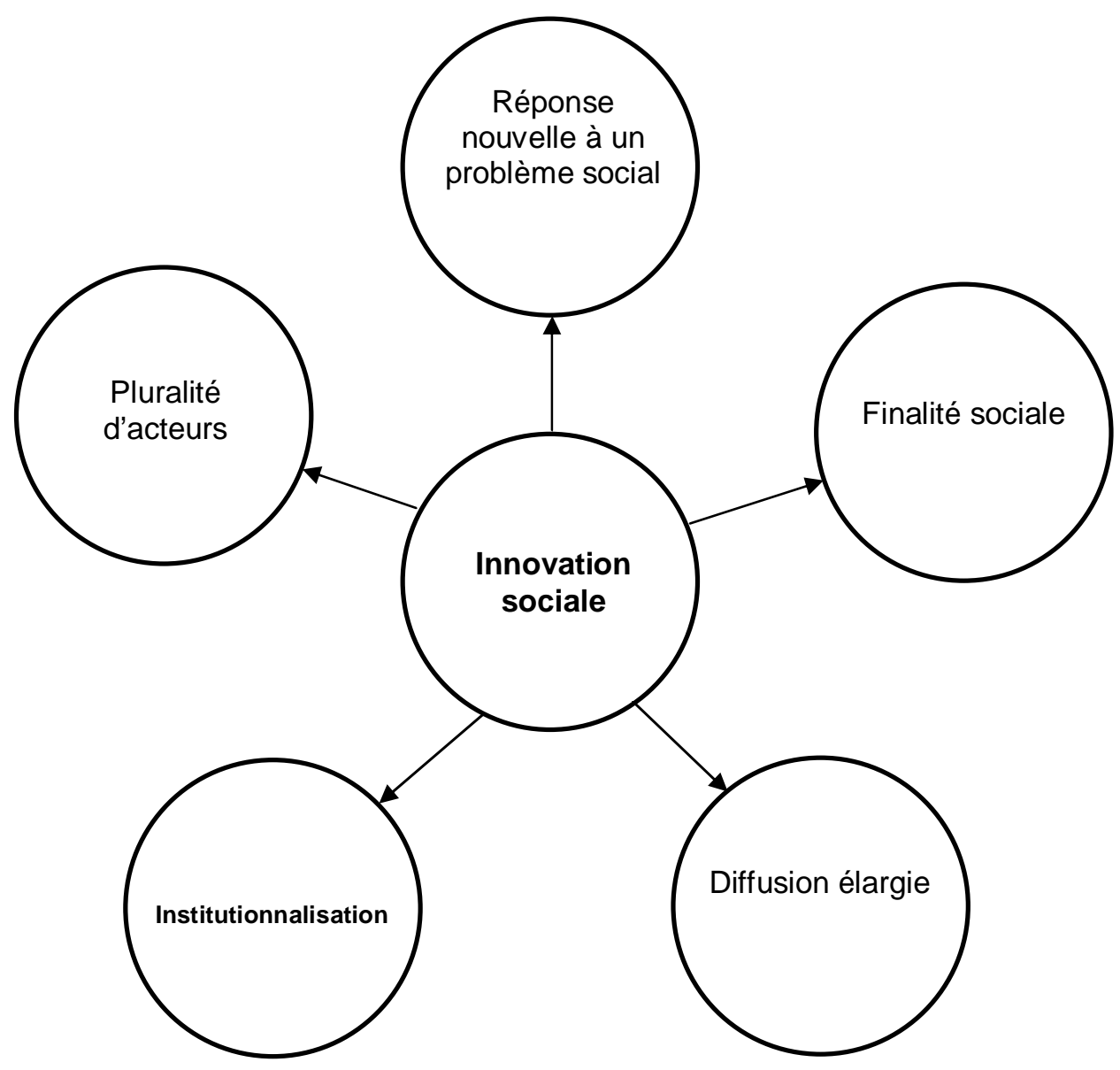


Une innovation sociale consiste en une réponse nouvelle à un problème social, une réponse poursuivant une finalité sociale et impliquant plusieurs acteurs. En plus de son adoption élargie, elle doit être institutionnalisée en devenant la pratique normative courante.

À la lumière de ce cadre d'analyse, une innovation sociale consiste en une réponse nouvelle à un problème social, une réponse poursuivant une finalité sociale et impliquant plusieurs acteurs. En plus de son adoption élargie, elle doit être institutionnalisée en devenant la pratique normative courante.

Premièrement, une innovation sociale est une réponse nouvelle à un problème social concret, une situation jugée inacceptable ou insatisfaisante, vécue le plus souvent localement. Elle se développe sous une forme peu codifiée, non linéaire et en heurt souvent avec l'ordre organisationnel qui implique alors une transgression des règles établies ${ }^{7}$. Deuxièmement, au-delà de la réponse à un besoin concret vécu localement, les innovateurs poursuivent une finalité sociale et s'appuient sur des valeurs et des aspirations sous-jacentes à leur action. Troisièmement, l'innovation sociale est un processus où, dès le départ, des pionniers proposent des idées nouvelles et transigent avec d'autres acteurs plus ou moins réceptifs à leur mise à l'essai ${ }^{8}$. Pour parler d'innovation sociale, on retrace une «appropriation» de l'innovation par la communauté locale issue de la négociation et du compromis. Elle peut aller jusqu'à émerger d'une co-construction impliquant divers acteurs locaux. Quatrièmement, pour porter son nom, une innovation sociale doit connaitre une certaine diffusion et être adoptée en dehors de son cadre initial. Elle doit donc avoir démontré son efficacité ou avoir reçu un jugement extérieur positif. Enfin, l'institutionnalisation constitue l'étape ultime du processus d'innovation sociale. Elle renvoie à une pérennisation qui fait en sorte que l'innovation s'inscrit dans la durée, ce qui implique souvent une intervention de l'État. L'innovation s'inscrit donc dans une dialectique incluant, d'une part, une rupture avec l'institution, mais d'autre part une construction de l'institution en devenant la nouvelle norme qui sera à son tour éventuellement défiée.

Une innovation sociale doit connaître une certaine diffusion et être adoptée en dehors de son cadre initial.

\section{DIX INITIATIVES DE L'OUTAOUAIS SOUS LA LOUPE DE L'INNOVATION SOCIALE}

Parmi une cinquantaine de pratiques sociales jugées créatives et ayant obtenu un succès reconnu en Outaouais, dix cas ont fait l'objet de l'étude au moyen d'une démarche appuyée par la recherche documentaire, un questionnaire et des entrevues semi-dirigées. L'échantillon de recherche est un échantillon non probabiliste raisonné associé à une conception élargie du terme « initiatives sociales » non limité à l'univers de la santé et des services sociaux. Les dix cas retenus l'ont été à partir des critères suivants : 1. initiatives perçues en Outaouais comme étant des succès ou comportant une bonne part de réussite; 2 . initiatives mises en œuvre depuis au moins trois ans; 3. initiatives des milieux urbains et des milieux ruraux et
4. initiatives provenant du secteur public et du secteur communautaire. Les dix initiatives sont les suivantes :

- Le Centre d'intervention en abus sexuels pour la famille (CIASF);

- Le LAB. Programme d'expression artistique pour les 16 à 30 ans vivant des difficultés telles toxicomanie, itinérance, exclusion sociale, pauvreté, ou désirant s'exprimer autrement, une initiative du Centre d'intervention et de prévention en toxicomanie de l'Outaouais (CIPTO);

- La Relance Outaouais. Une entreprise d'économie sociale d'insertion;

- L'intervention de quartier de Gatineau. Cas du quartier Jean Dallaire-Front du secteur Hull et l'Inter-quartiers qui constitue l'organe fédératif 
de sept organisations de type maisons de quartier;

- Le Fonds de développement social de l'Agence de la santé et des services sociaux de l'Outaouais (Fonds DS);

- Les Logements intégrés de Hull. Appartements offrant des services de soutien à domicile 24 heures par jour et 7 jours par semaine aux personnes lourdement handicapées physiquement;

- Le Carrefour Jeunesse Emploi de l'Outaouais (CJEO);

- Le Dépanneur Sylvestre. Espace commercial axé sur la relation conviviale et l'intégration sociale et professionnelle accessible à tous sept jours sur sept. OBNL et coopérative de solidarité de sensibilisation en alimentation saine et de mobilisation d'entraide;

- Bouffée d'Oxygène (BO2). Programme du Service d'animation jeunesse de l'Outaouais (SAJO) qui offre en milieu scolaire des animateurs et des activités destinées aux adolescents;

- Le plan intégré de transport en commun de la Société de transport de l'Outaouais (STO).

Outre la recherche documentaire, la collecte de données a été effectuée auprès des organismes concernés à partir d'un seul informateur par organisme. La convergence entre les propos recueillis, la documentation des organismes ainsi que la consultation que nous avons faite des recherches menées antérieurement au sujet de plus de la moitié des initiatives de notre échantillon atténue cette limite de notre recherche en assurant un minimum de validation des points de vue exposés par les personnes interviewées.

\subsection{Des initiatives sises à diverses étapes du processus d'innovation sociale}

Le premier constat issu de notre étude renvoie à l'idée que l'innovation est un processus. Les projets aussi créatifs ou novateurs soient-ils empruntent des trajectoires exigeant du temps. En effet, l'innovation sociale prend forme d'un processus qui va de l'émergence d'une idée novatrice jusqu'à sa diffusion large qualifiée en son aboutissement d'institutionnalisation. Entre ces deux pôles, l'idée novatrice passe par diverses étapes et ce n'est qu'a posteriori que l'on pourra dire si l'on est en présence ou non d'une réelle innovation sociale. La durée de vie d'une innovation est de plus tributaire du contexte socio-historique dans lequel elle se déploie et ce qui était nouveau il y a un certain temps et dans un contexte social quelconque deviendra désuet à plus ou moins longue échéance. Selon certains chercheurs, deux destinées attendent paradoxalement l'innovation: le « cimetière » si elle n'est pas reproduite ou la « routinisation» si son institutionnalisation en fait une pratique courante fortement répandue ${ }^{7}$.

Tout au long du processus d'innovation, la diffusion revêt une importance stratégique. La diffusion n'est pas un concept monolithique et doit être entendue dans ses divers sens, soit faire connaître ou encore reproduire. D'une part, dans le langage courant ou professionnel, diffuser renvoie à la visibilité à donner à une idée ou une intervention. Dans ce premier sens diffuser veut dire faire connaître une idée créative ou une intervention novatrice. Dans le sens employé par plusieurs écrits en sociologie ou en économie, la diffusion renvoie à la reproduction ailleurs d'une pratique novatrice. La diffusion d'une innovation est donc indispensable pour que celle-ci voie son rayon d'action s'étendre au-delà du milieu où elle a été originellement expérimentée. Nous avons étudié les pratiques outaouaises en analysant dans quelle mesure elles se sont faites connaître dans la région et hors de la région. Nous avons aussi étudié dans quelle mesure ces pratiques novatrices ont trouvé preneur ailleurs soit dans des milieux qui les ont adoptées en les reproduisant ou en ont fait une source d'inspiration pour renouveler leurs pratiques. Nous parlerons alors d'appropriation régionale ou extra régionale. Finalement, nous avons constaté que certaines pratiques ont fait l'objet d'une reproduction ou adaptation systématique et largement territorialisée. On dira de celles-ci qu'elles ont fait l'objet d'institutionnalisation, étape ultime de la diffusion au sens de la reproduction.

Parmi les dix cas étudiés en Outaouais, on retrace des initiatives à diverses étapes du processus innovant. Quatre en étaient à l'émergence avec la jeunesse de leur mise en œuvre de 
moins de cinq ans. Parmi celles affichant une vitesse de croisière empreinte de stabilité, la diffusion au-delà du territoire local variait depuis une diffusion régionale jusqu'à une diffusion large en terme de connaissance des projets sans que cela ne signifie nécessairement que les pratiques ont été répliquées ailleurs. Selon les résultats obtenus à partir de l'échantillon étudié et conformément à la littérature sur le degré d'appropriation ${ }^{7}$, l'initiative de l' «intervention de quartier de Gatineau » a suivi un processus innovant avec une appropriation de proximité, alors que celle du Carrefour Jeunesse Emploi de l'Outaouais a connu une appropriation étendue. L'appropriation des initiatives par le milieu ou une partie du milieu était observable dans deux cas même si on ne pouvait parler d'institutionnalisation au sens classique du terme. Deux cas seulement se situent au bout du processus en tant que pratiques institutionnalisées, l'une ayant été clairement à l'origine d'une reproduction à l'ensemble du Québec et l'autre ayant fait partie des quelques pratiques simultanément inspiratrices de programmes qui ont rapidement émergé au Québec. Le tableau qui suit situe les 10 cas étudiés en fonction de leur stade de diffusion.

Tableau 1 : Les innovations sociales en Outaouais en fonction de leur stade de diffusion

\begin{tabular}{|lcccc|}
\hline $\begin{array}{l}\text { Émergence } \\
\text { diffusion/visibilité } \\
\text { restreinte }\end{array}$ & $\begin{array}{c}\text { diffusion/visibilité } \\
\text { large }\end{array}$ & $\begin{array}{c}\text { Appropriation } \\
\text { régionale/extrarégionale }\end{array}$ & Institutionnalisation \\
\hline $\begin{array}{l}\text { Dépanneur Sylvestre } \\
\text { Le LAB }\end{array}$ & CIASF & plan intégré STO & Intervention quartier/Interquartier & CJEO \\
$\begin{array}{l}\text { Bouffée d'oxygène } \\
\text { Fonds DS }\end{array}$ & & Logements intégrés & La Relance \\
\end{tabular}

\section{Une initiative novatrice prendra le nom d'innovation sociale lorsqu'elle est réussie en phase d'expérimentation puis reproduite ailleurs pour enfin être reconnue, diffusée et dotée de moyens permettant sa consolidation comme pratique.}

Avec le recul et l'analyse du cheminement ou du processus, une initiative novatrice prendra le nom d'innovation sociale lorsqu'elle est réussie en phase d'expérimentation puis reproduite ailleurs pour enfin être reconnue, diffusée et dotée de moyens permettant sa consolidation comme pratique. Cette labellisation de l'innovation sociale ne signifie pas pour autant que le caractère novateur d'une pratique ne prend forme qu'en son aboutissement à grande échelle. En distinguant l'innovation sociale micro de l'innovation sociale macro, les transformations organisationnelles micro des transformations institutionnelles macro ${ }^{12}$, le
Carrefour jeunesse emploi de l'Outaouais a franchi tout le processus d'innovation sociale, a connu un processus d'appropriation fort et a connu une diffusion large menant à son institutionnalisation au Québec. Cette initiative a atteint un niveau de reconnaissance sociale et de légitimité suffisant pour que l'on puisse parler d'innovation sociale. Les 10 cas de notre étude ont généré des réponses originales, nouvelles, inédites, à un problème social particulier.

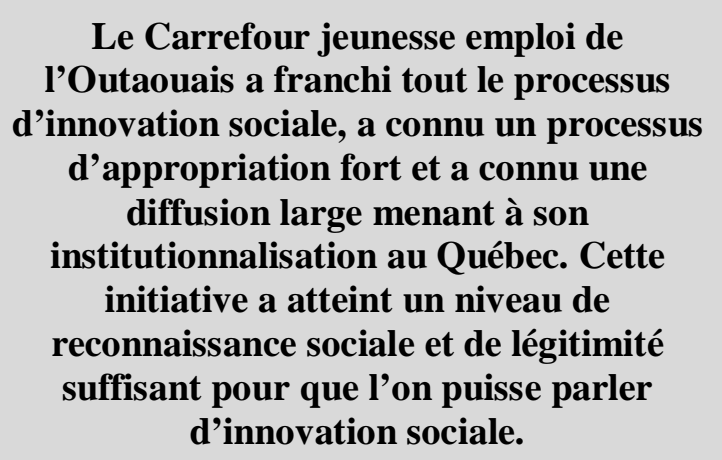

Le Carrefour jeunesse emploi de l'Outaouais a franchi tout le processus d'innovation sociale, a connu un processus d'appropriation fort et a connu une diffusion large menant à son institutionnalisation au Québec. Cette initiative a atteint un niveau de reconnaissance sociale et de légitimité suffisant pour que l'on puisse parler d'innovation sociale. 
Par contre, certaines des pratiques novatrices n'ont pas réussi à ce jour à franchir les frontières de l'Outaouais. Parmi les 10 initiatives étudiées, rares sont celles qui ont connu un processus d'appropriation au sens fort du terme. Elles ont reçu l'appui d'institutions locales et jouissent d'une bonne reconnaissance au palier local, sans pour autant qu'elles aient reçu cette reconnaissance plus élargie qui ferait qu'elles seraient adoptées en dehors de leur contexte initial, atteignant ainsi un plus haut degré de généralité. Leur diffusion au sens de la reproduction ailleurs ne semble pas avoir été suffisante pour qu'elles deviennent des modèles innovants au niveau macro. Ce constat conduit à explorer les conditions favorables et les conditions défavorables à l'innovation sociale.

\section{LES CONDITIONS FAVORISANT OU NON L'INNOVATION SOCIALE}

L'émergence d'idées novatrices n'entraîne pas automatiquement la mise en œuvre de pratiques renouvelant la réponse à des problèmes sociaux tout comme l'expérimentation locale réussie n'est pas garante de sa reproduction à large échelle. En analysant les initiatives outaouaises, nous avons pu dégager des facteurs qui sont de nature à favoriser ou à freiner l'innovation sociale à un moment ou l'autre du processus de son déploiement. Sans prétendre qu'ils sont les seuls en cause, nos données confirment leur importance. Selon les recherches dans le domaine, il ne suffit pas que des besoins nouveaux soient laissés sans réponse pour que naisse l'innovation, mais bien que des gens se mobilisent pour y répondre ${ }^{12}$. En effet, «l'environnement produit des occasions qui peuvent ou non être saisies ». La centralité des acteurs dans le processus d'innovation sociale est indubitable. Encore faut-il que leur soient donnés les moyens de mener à terme les projets émergents et que des conditions minimales de réceptivité soient en place dans les milieux concernés. Ces conditions minimales, présentées à la figure 2, sont multiples et méritent d'être précisées.

Figure 2 : Facteurs favorisant l'innovation sociale

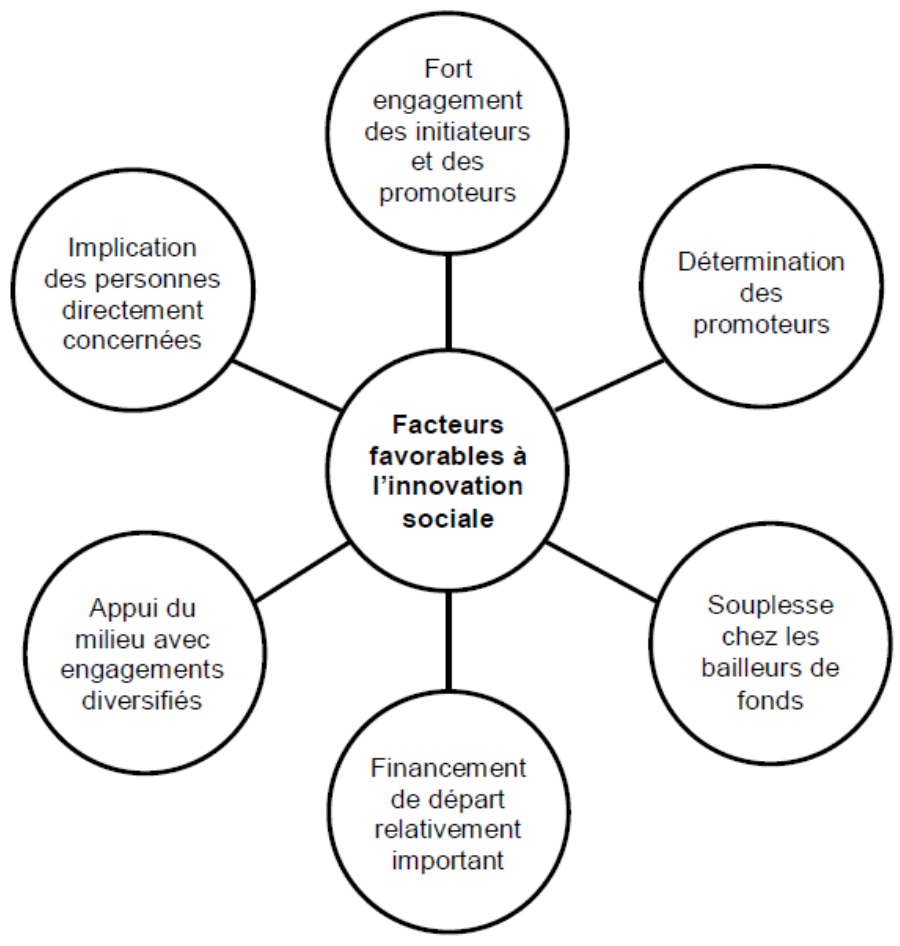




\section{Le fort engagement des initiateurs et des} promoteurs est le premier de ces facteurs favorisant l'innovation sociale. La très grande majorité des cas étudiés ont comme promoteurs des personnes qui croient fortement en leur action et qui apparaissent des gens passionnés de leur projet.

Le fort engagement des initiateurs et des promoteurs est le premier de ces facteurs favorisant l'innovation sociale. La très grande majorité des cas étudiés ont comme promoteurs des personnes qui croient fortement en leur action et qui apparaissent des gens passionnés de leur projet. La détermination des promoteurs s'avère ensuite un facteur clé pour franchir le stade de l'émergence de l'idée et passer à sa mise à l'essai. Les entrevues menées témoignent de la persévérance des promoteurs à défendre le projet et à passer à l'action malgré la résistance souvent rencontrée. Cette persévérance va parfois jusqu'à prendre le risque de contourner des règles en place. Garder le cap sur un projet novateur s'apparente parfois à un geste déviant et à la transgression de l'ordre établi'. La souplesse chez les bailleurs de fonds est non négligeable pour que des idées novatrices aient les moyens de voir le jour. Les idées nouvelles souvent en rupture avec les pratiques antérieures se retrouvent le plus souvent hors normes dans les programmes de financement de tout acabit. La souplesse de bailleurs de fonds qui acceptent sous la pression des promoteurs de «travailler hors norme» ou de modifier leurs propres règles devient un atout pour la progression d'idées nouvelles. Nos données ont montré que des gestionnaires se sont laissés convaincre du bien fondé de certaines initiatives et dans certains cas sont même devenus des alliés des promoteurs. Cette souplesse est essentielle lors d'un premier financement d'idées novatrices et s'avère aussi utile à d'autres étapes pour faire en sorte qu'une pratique réussie dépasse le territoire local.

Un financement de départ relativement important vient ensuite donner aux promoteurs les conditions nécessaires pour se concentrer sur l'action et le développement des projets plutôt que sur le bricolage financier récurrent. Un financement consistant au stade de l'émergence donne le moyen de mettre en place les conditions permettant à un projet de réussir s'il est bien construit et s'il répond à des besoins en proposant des solutions renouvelant les pratiques. Le même projet démarré sans moyens consistants serait souvent voué à l'échec. L'implication des personnes directement concernées par les problèmes sociaux auxquels les projets s'attaquent se présente comme un facteur favorable au développement d'une innovation dans plusieurs des cas étudiés.

\section{L'implication des personnes directement concernées par les problèmes sociaux auxquels les projets s'attaquent se présente comme un facteur favorable au développement d'une innovation.}

La présence active et la prise de parole des populations concernées suscitent la délibération autour des projets qualifiés de novateurs. La réponse qui émergera de cette délibération aura plus de chances d'être adéquate considérant les besoins et les intérêts des gens et des milieux où se déploient les projets. Enfin, l'appui du milieu sous forme d'engagements diversifiés est un facteur important pour soutenir les initiatives qui franchissent le cap de l'expérimentation pour amorcer la diffusion de l'innovation. Selon les cas, cet appui du milieu est financier, technique, politique ou encore prend la forme du soutien populaire dans la communauté.

\section{L'appui du milieu sous forme d'engagements diversifiés est un facteur important pour soutenir les initiatives qui franchissent le cap de l'expérimentation pour amorcer la diffusion de l'innovation.}

Si certains facteurs favorisent l'innovation, l'étude en a aussi identifié d'autres qui freinent l'innovation. Nos données indiquent quatre obstacles (illustrés à la figure 3) qui, en Outaouais, sont rapportés comme les principaux facteurs faisant freins à l'innovation. Deux relèvent des attitudes ou comportements des acteurs face à la nouveauté. Deux sont liées à l'attribution de ressources financières au cours du processus d'innovation sociale. 
Figure 3 : Les freins à l'innovation sociale

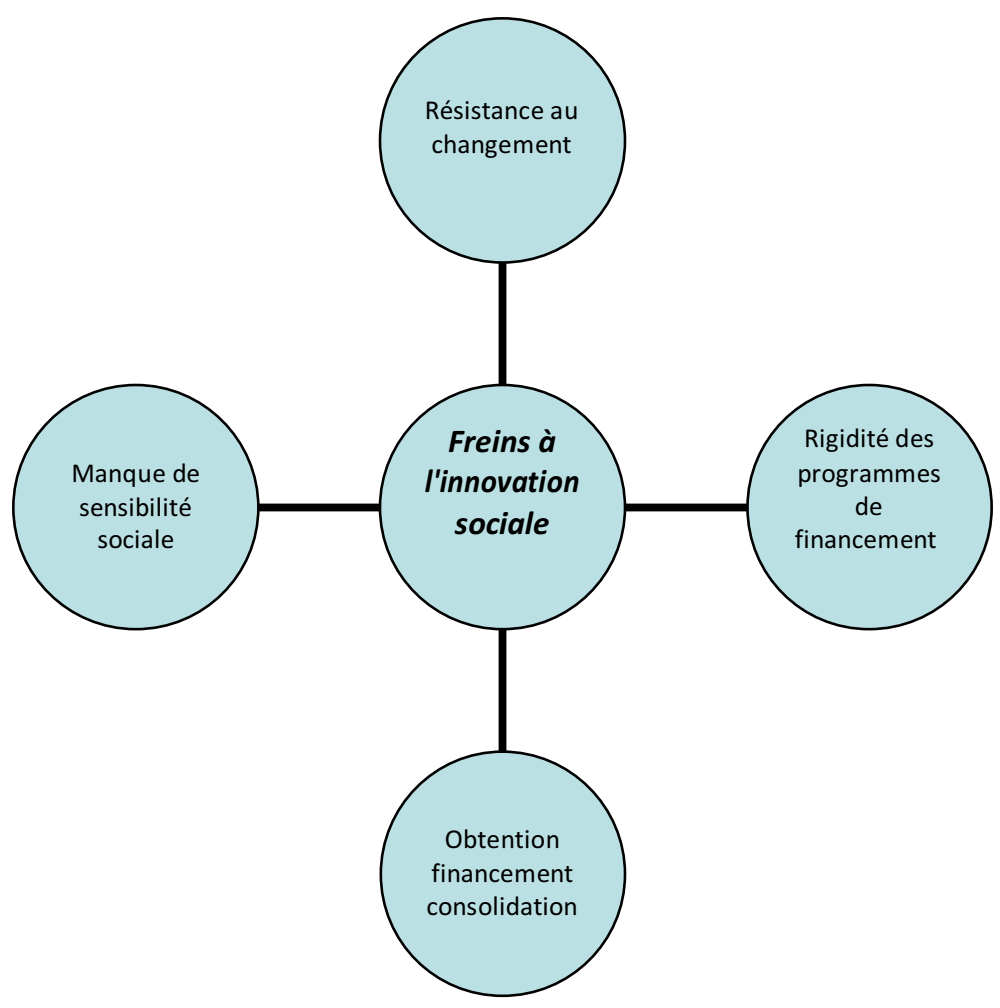

La résistance au changement a été identifiée comme l'un des freins les plus fréquents à l'innovation. La majorité des études se penchant sur le changement évoque cette résistance au changement qui prend diverses formes. Les travaux traitant des valeurs évoquent la résistance au changement. Les travaux en gestion de ressources humaines évoquent la résistance aux changements dans les organisations. Les travaux en prévention sociale et en organisation communautaire évoquent la résistance au changement. On ne s'étonnera pas de sa présence au cours du processus d'innovation sociale invitant à la modification de pratiques sociales. Elle est souvent liée à la confrontation entre des acteurs porteurs de valeurs parfois éloignées les unes avec les autres et à la remise en question de pratiques antérieures portées par des acteurs convaincus de leur pertinence. La résistance au changement provient parfois aussi de la méconnaissance des projets, parfois de la crainte de la perte des acquis chez des intervenants, parfois du conservatisme des individus, de groupes dans la communauté ou de populations.

\section{La résistance au changement a été identifiée comme l'un des freins les plus fréquents à l'innovation.}

Le manque de sensibilité sociale face à certains problèmes sociaux est un autre frein à l'innovation sociale. Certains problèmes sociaux entraînent plus que d'autres un réflexe de sympathie dans la population. Les causes qualifiées d'humanitaires reçoivent plus d'attention que les causes prônant le développement local par exemple. Le soutien aux victimes génère souvent plus de sympathie que l'intervention auprès des agresseurs ou des déviants. On pourrait dire ironiquement que certains problèmes sociaux sont plus populaires que d'autres. Lorsque des intervenants veulent s'attaquer à des problèmes dont le capital de 
sympathie ou la popularité est faible ou encore s'attaquer à des situations de l'ordre du tabou, on a tendance à faire peu de cas de leurs idées nouvelles peu importe la gravité des problèmes concernés. Dans ces cas, il est difficile de recueillir l'adhésion du milieu, le soutien financier requis et les appuis politiques nécessaires à la progression d'une initiative novatrice.

\section{La rigidité des programmes gouvernementaux de financement est souvent nommée comme un frein majeur à l'innovation.}

La rigidité des programmes gouvernementaux de financement est souvent nommée comme un frein majeur à l'innovation. Nos interlocuteurs ont évoqué dans cette veine le caractère «structuré et structurant» des programmes, le financement compartimenté qui va à l'encontre d'une approche globale, le financement fragmenté, la nécessité de devoir contourner les programmes normés, la gestion rigide et les attitudes fermées de fonctionnaires. Bref, presque tous se plaignent que leur projet n'entre dans aucune des cases des différents programmes. Mais... au fond, n'est-ce pas là une caractéristique de l'innovation sociale que de n'entrer dans aucun programme établi?

Enfin, la difficulté à obtenir du financement de consolidation des initiatives mises sur pied est un obstacle à ne pas négliger. S'il semble qu'il soit relativement facile de trouver un financement de démarrage, il semble qu'il soit plus difficile d'y avoir accès pour consolider les activités après la phase d'expérimentation. Parmi les cas étudiés en Outaouais, nous avons trouvé quelques exemples éloquents d'organisations ou de programmes novateurs qui ont survécu au moyen de bricolages financiers et de stratégies de «débrouille». Si les projets nouveaux ont de la difficulté à financer le stade de la consolidation locale, on peut imaginer que peu d'énergies seront consacrées à faire connaître ailleurs leur réussite, autre obstacle à la diffusion de l'idée novatrice.

Les facteurs favorables ou défavorables à l'innovation sociale expliquent en partie les rythmes d'évolution plus ou moins lents des idées novatrices, leur transformation en expérimentation documentée, l'adhésion plus ou moins forte d'acteurs diversifiés aux changements de pratiques conséquents aux innovations proposées. Nos données indiquent que ces facteurs n'expliquent cependant pas tout. En effet, l'action des innovateurs, tant dans le milieu communautaire qu'en milieu institutionnel, est ponctuée de défis tels que: doter une initiative des moyens de se consolider, faire de la diffusion un enjeu envisagé dès la phase d'expérimentation d'une idée ou d'un projet novateurs, concevoir diverses stratégies favorisant l'appropriation d'une nouvelle pratique par les gens concernés par le problème et par les gens détenant l'influence voulue pour faire connaître la valeur des solutions nouvelles expérimentées. Dans cette étude, le défi de la diffusion est ressorti comme enjeu prédominant pour la valorisation des succès ou innovations sociales en Outaouais.

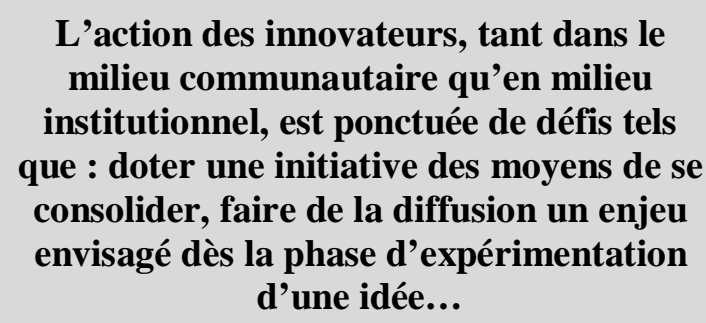

\section{PASSER DE L'INVENTION À L'INNOVATION SOCIALE : LE DÉFI DE LA DIFFUSION}

La distinction entre pratique novatrice et innovation sociale nous incite à examiner pourquoi les pratiques novatrices et inédites que l'on trouve en Outaouais parviennent difficilement à franchir les frontières de la région et à dépasser le stade de "l'invention». La question de la diffusion est ici centrale. Parmi les explications sous-jacentes au défi de la diffusion de l'innovation sociale: le pouvoir d'attraction d'une innovation, un contexte social opportun, les ressources requises, le soutien de la recherche et le soutien des acteurs économiques et 
politiques s'avèrent des déterminants d'une importance capitale et dont il convient de préciser leur portée.

\section{Pouvoir d'attraction d'une innovation}

Certaines pratiques sociales, même si elles sont inédites, ne franchiront jamais le stade de l'innovation sociale tout simplement parce qu'elles ne suscitent pas suffisamment d'intérêt. Autrement dit, on ne s'intéresse pas aux nouvelles pratiques si celles-ci n'ont pas démontré leur efficacité, d'où l'importance de leur expérimentation et de la diffusion des résultats des expérimentations. Il va sans dire que les expériences jugées des réussites seront plus attrayantes que celles qui demeurent quasi inconnues si novatrices soient-elles. Nous savons également que des pratiques sociales novatrices n'atteignent peut-être pas le stade de l'innovation parce qu'elles font appel à des changements de pratiques ou de valeurs trop importants et, de ce fait, rencontrent une forte résistance ce qui approche la théorie des «paliers institutionnels ${ }^{9}$.

\section{Contexte social opportun}

Une autre raison peut expliquer le peu d'écho que trouve une initiative en dehors de son lieu d'origine. En effet, des pratiques novatrices ne sont pas reprises ou reproduites dans d'autres milieux simplement parce que les conditions requises ne sont pas présentes dans ces milieux. Ainsi, un milieu donné peut vivre un problème social identique à un autre, souhaiter s'inspirer d'une pratique développée ailleurs, mais ne pas être capable de mobiliser les ressources humaines ou financières nécessaires. La diffusion d'une innovation dépend aussi de son « timing », c'est-à-dire de « la coïncidence entre le projet novateur et les capacités organisationnelles d'une collectivité ${ }^{12}$. On peut faire l'hypothèse que plus une pratique novatrice nécessite le concours d'un nombre important d'acteurs et la mobilisation de ressources variées, plus il sera difficile de la reprendre dans un autre milieu.

\section{Accès aux ressources requises}

Le manque de ressources peut expliquer au moins en partie cette difficulté à diffuser. Il faut considérer également le fait que la diffusion d'une expérience exige des compétences qui ne sont pas les mêmes que celles qui sont requises pour assurer son développement. Or, les innovateurs dans le secteur du développement social et à plus forte raison dans le secteur communautaire sont souvent avant tout des bâtisseurs, qui mettent peu d'énergie à développer les compétences requises ou à développer de l'intérêt pour entrer dans des opérations centrées sur la diffusion. On peut également se demander si l'innovation sociale n'a pas aussi besoin d'un petit coup de main pour assurer sa diffusion. L'innovation technologique est propulsée par la logique de marché et dispose de moyens qu'on ne retrouve pas dans le secteur non marchand et de façon encore plus évidente dans les organisations du secteur communautaire.

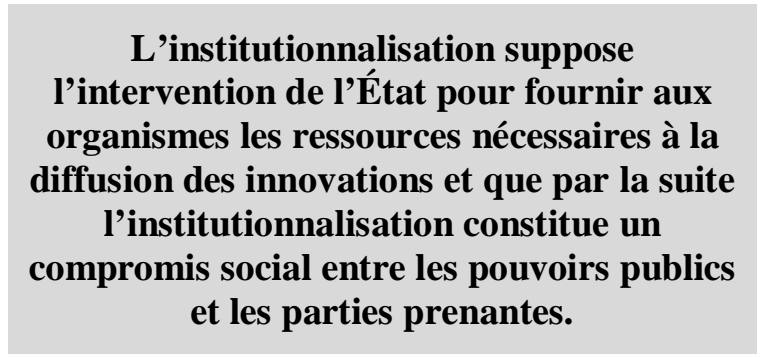

Selon Lévesque, l'institutionnalisation suppose l'intervention de l'État pour fournir aux organismes les ressources nécessaires à la diffusion des innovations et que par la suite l'institutionnalisation constitue un compromis social entre les pouvoirs publics et les parties prenantes $^{2}$. Dans le cas de l'Outaouais, plusieurs pratiques novatrices ont vu le jour dans le secteur communautaire ce qui accroît le défi de se doter des moyens de la diffusion. Faire connaître ses pratiques exige d'y consacrer des ressources. Or, nous avons constaté que les promoteurs de nouvelles pratiques consacrent leurs énergies d'abord et avant tout à la mise en œuvre de leur projet local puis à sa consolidation. Leur rayon d'influence est d'abord local et dans certains cas régional. Camper l'action dans un contexte plus large exige de voir au-delà des effets immédiats des actions ou projets entrepris, et dès le point de départ, de songer au caractère d'exemplarité du projet et à sa capacité d'être reproduit ou adapté ailleurs. La recherche peut ici jouer un rôle intéressant. 


\section{Soutien de la recherche}

Le lien entre diffusion et recherche est connu sous l'angle de l'effort consenti à la diffusion des résultats de recherche via les canaux classiques ou ceux du transfert des connaissances qui élargissent la portée de ces résultats. L'association recherche et diffusion de l'innovation a cependant été moins explorée. Une étude de 307 projets en prévention sociale dans les années quatre-vingt-dix soulignait que parmi les facteurs expliquant la réussite de projets ou de programmes dont plusieurs étaient novateurs, l'on retrouve le soutien de la recherche ${ }^{11}$.

\section{Certains grands organismes}

subventionnaires amorcent une stratégie de soutien à la recherche dans la perspective du soutien à l'innovation comme au

Canada le CRSH qui a initié les alliances

de recherche université-milieu (ARUC) où chercheurs et partenaires sociaux se côtoient dans un processus de coconstruction de la recherche et de sa diffusion.

Certains grands organismes subventionnaires amorcent une stratégie de soutien à la recherche dans la perspective du soutien à l'innovation comme au Canada le CRSH qui a initié les alliances de recherche université-milieu (ARUC) où chercheurs et partenaires sociaux se côtoient dans un processus de co-construction de la recherche et de sa diffusion. Dans notre étude en Outaouais, on remarque que plusieurs projets ont été l'objet de recherches à un moment ou l'autre de leur évolution. C'est particulièrement le cas du Carrefour jeunesse emploi $^{13}$ et de l'intervention de quartier ${ }^{10}$, qui ont connu une large diffusion à l'échelle québécoise dans le cas du CJEO et à l'échelle régionale dans l'autre cas. Le lien entre la recherche et l'initiative novatrice constitue une piste à approfondir pour expliquer la diffusion plus ou moins forte de certaines innovations.

\section{Soutien des acteurs économiques et politiques}

La diffusion est aussi apparentée à l'appropriation d'une innovation par différents acteurs. Déjà l'adhésion des gens concernés par le problème à solutionner est, comme mentionné préalablement, un atout dans la mise à l'essai d'une solution novatrice. Il faut cependant élargir le cercle des adhérents au projet pour que celui-ci prenne de l'expansion. Les projets novateurs qui trouvent un ancrage dans un terreau collectif ont de meilleures chances de se rendre à bon port puis de se diffuser. Ils peuvent trouver un ancrage dans une organisation publique ou parapublique. C'est le cas de l'intervention de quartier longtemps portée et animée par l'organisation communautaire du CLSC de Hull qui, par la suite, a essaimé en dehors du quartier d'origine pour s'étendre dans le territoire métropolitain portée par l'Interquartier. Les projets peuvent s'insérer dans la dynamique d'un réseau ou d'un organe fédératif, comme c'est le cas de Bouffée d'oxygène de SAJO membre de la Fédération québécoise des centres communautaires de loisir. Ils peuvent aussi être soutenus et/ou adoptés par des mouvements sociaux comme dans le domaine de l'habitat. L'ancrage des projets dans un réseau favorise leur prise en compte par les milieux où se trouvent des gestionnaires et décideurs et par des élus détenant un pouvoir d'influence. Le réseautage d'intervenants sociaux ou d'organisations dans le secteur social au sens large s'avère souvent un milieu où les participants partagent les mêmes convictions et défendent les mêmes causes. L'essaimage des innovations a donc plus de chances de s'avérer large si les réseaux d'appartenance ne se limitent pas à ceux qui sont en place à l'échelle régionale.

L'action politique en lien avec la diffusion

de l'innovation semble avoir été peu activée dans les projets étudiés sauf dans le cas

évident du Carrefour jeunesse emploi qui s'est répandu comme pratique à la suite d'une action au niveau du bureau du premier ministre du Québec.

L'action politique en lien avec la diffusion de l'innovation semble avoir été peu activée dans les projets étudiés sauf dans le cas évident du Carrefour jeunesse emploi qui s'est répandu comme pratique à la suite d'une action au niveau du bureau du premier ministre du 
Québec et à un niveau plus local dans le cas de l'intervention de quartier où la ville de Gatineau soutient en partie de l'expansion de cette pratique dans divers quartiers. Même si les innovations jouissent généralement d'une assez bonne reconnaissance au niveau des institutions locales, nos interlocuteurs identifient des carences dans l'appropriation des innovations par les acteurs et les décideurs locaux. On déplore des faiblesses dans le soutien des institutions locales et dans les partenariats nécessaires avec les innovateurs pour la construction de ce qui deviendra, le cas échéant, une innovation sociale. L'aspect politique de la diffusion de l'innovation sociale est une dimen- sion où l'action doit s'immiscer dans des espaces d'interaction entre l'État, les mouvements sociaux, les groupes de pression, les collectivités locales, entre le privé et le public et le communautaire.

\section{L'aspect politique de la diffusion de} l'innovation sociale est une dimension où l'action doit s'immiscer dans des espaces d'interaction entre l'État, les mouvements sociaux, les groupes de pression, les collectivités locales, entre le privé et le public et le communautaire.

\section{CONCLUSION : DEUX QUESTIONS SOUMISES À LA RÉFLEXION}

L'étude de 10 initiatives à succès en Outaouais laisse voir que la créativité des intervenants s'active souvent pour trouver des réponses nouvelles à des problèmes sociaux qui évoluent. Lorsque soutenue par des conditions favorables et lorsque déployée dans un contexte social, économique et politique propice, la créativité des intervenants peut entraîner la mise à l'essai de pratiques novatrices. Si les expérimentations réussies débordent des milieux où elles ont été mises à l'essai, dans le cadre conceptuel actuel de l'innovation sociale, elles peuvent entrer dans une phase de diffusion et en arriver à l'institutionnalisation. À la suite de cette recherche, il nous semble approprié de poser deux questions, l'une sur le soutien à l'innovation, l'autre sur la façon de nommer les avancées novatrices dans le secteur du développement social des communautés.

Lorsque soutenue par des conditions favorables et lorsque déployée dans un contexte social, économique et politique propice, la créativité des intervenants peut entraîner la mise à l'essai de pratiques novatrices.

D'abord, quels sont les éléments à activer dans les organisations, les collectivités locales et les territoires régionaux pour soutenir l'innovation dans le champ du développement social au
Québec? La question en entraîne d'autres. Yaurait-il lieu de songer à des fonds de soutien à l'innovation? Y-aurait-il lieu de penser à de la formation sur la pensée créative? Y-aurait-il lieu de mettre en place des cercles d'innovation? Des programmes régionaux d'innovation pourraientils élargir le registre des programmes de soutien au développement social?

L'innovation sociale traverse la littérature scientifique, le discours des entreprises d'économie sociale, celui d'une partie des organismes communautaires et le discours politique. La littérature actuelle laisse entendre que le processus est long et assez complexe pour que des projets novateurs en arrivent à se qualifier d'innovations sociales au sens achevé du terme. Pourtant, la volonté de transformation des pratiques sociales n'a pas cessé de s'activer chez les intervenants et les expérimentations réussies se comptent en bon nombre. N'aurait-il pas lieu de réintroduire l'idée du renouvellement des pratiques sociales du moins à l'échelle de l'étude des pratiques de développement social d'un milieu ou d'un territoire donné. Cela permettrait d'identifier les éléments d'amélioration et de renouvellement des pratiques en réintroduisant la valeur de renouvellement à l'échelle d'une organisation, d'une collectivité locale ou d'une région. Parler de renouvellement des pratiques sociales ne vient pas menacer l'étude par la suite des pratiques novatrices pour déterminer le 
degré d'adoption à large échelle des innovations et leur institutionnalisation.

Dans le domaine social, il appert que les innovateurs résistent au discours du bien-fondé de l'institutionnalisation. Les organismes communautaires ont souvent une sensibilité forte aux carences des services ou des pratiques fortement institutionnalisés, laissant peu de marge de manœuvre pour les populations vulnérables souvent atypiques et moins bien desservies par les institutions publiques. Il y a lieu de réfléchir sur le fait que des pratiques novatrices peuvent participer à l'innovation sociale sans nécessairement se transformer en innovations institutionnalisées ${ }^{4}$. Ainsi, leur diffusion ne s'opère pas par reproduction sous forme de copies mais par reproduction en termes d'adaptation soumises à un processus démocratique déterminant l'adhésion des groupes auxquelles ces innovations s'adressent.

Ces questions cherchent à relancer le débat sur la façon de qualifier les pratiques novatrices, sur le soutien à leur apporter, sur la façon de nommer les interventions axées sur le changement de pratiques. Bref, renommer l'introduction de la nouveauté dans les interventions et revisiter le renouvellement des pratiques sociales avant de mettre en place un modèle contribuant à la transformation des rapports sociaux où des interventions en faveur du développement social constituent des tentatives à explorer!

\section{BIBLIOGRAPHIE}

${ }^{1}$ Bourque, D., Proulx, J. et Fréchette, L. (2007). «Innovations sociales en Outaouais ». Cahier de l'ARUC-ISDC, Série recherches no 13, UQO, 52 pages.

${ }^{2}$ Lévesque, B. (2007). «L'innovation dans le développement économique et le développement social », Juan-Luis Klein et Denis Harrisson (dir.), L'innovation sociale. Émergence et effets sur la transformation des sociétés, Québec, PUQ, p. 43-70.

${ }^{3}$ Lévesque, B. (2005). «Innovations et transformations sociales dans le développement économique et le développement social : approches théoriques et politiques publiques », Cahiers du
CRISES, Coll. «Études théoriques », UQAM, 72 pages.

${ }^{4}$ Castel, O. et Prades, J. (2004). La vulnérabilité comme source de l'innovation sociale au Nord et au Sud. Conférence, 4e Rencontres du Réseau universitaire d'économie sociale et solidaire, CNAM Paris, 14 et 15 avril.

${ }^{5}$ Bouchard, C. (1999). «Recherche en sciences humaines et sociales et innovations sociales. Contribution à une politique de l'immatériel ", Québec, CQRS.

${ }^{6}$ Rollin, J. (2007). Acteurs et processus d'innovation sociale au Québec. Réseau Québécois en innovation sociale (RQIS), 77 pages.

${ }^{7}$ Comeau, Y. et coll. (2006). «Axe 2 - Conditions de vie », Juan-Luis Klein et Denis Harrisson (dir.), L'innovation sociale. Émergence et effets sur la transformation des sociétés, Québec, PUQ, p. 361-376.

${ }^{8}$ Assogba, Y. (2007). Le dépanneur Sylvestre. Une coopérative multifonctionnelle dans l'Outaouais. Cahier de l'ARUC-ISDC, Série Recherches, numéro 8,46 pages.

${ }^{9}$ Harrisson, D. et Klein, J.-L. (2007). «Introduction», Juan-Luis Klein et Denis Harrisson (dir.), L'innovation sociale. Émergence et effets sur la transformation des sociétés, Québec, PUQ, p. 1-14

${ }^{10}$ Favreau, L. et Fréchette, L. (2003) Organisation communautaire et intervention de quartier en Outaouais: l'expérience du quartier Jean Dallaire de Hull. Cahier Céris, série Recherches no 23, UQO, Gatineau, 43 pages.

${ }^{11}$ Fréchette, L. (2000). «La prévention une incontournable en psychologie communautaire». Agir au coeur des communautés: la psychologie communautaire et le changement social, Québec, Presses de l'Université Laval, p 217-248.

${ }^{12}$ Petitclerc, M. (2003). Rapport sur les Innovations et les transformations sociales, Cahiers du CRISES, Collection Études théoriques, CRISES, UQAM, 41 pages.

${ }^{13}$ Assogba, Y. (2000). « Organisation communautaire et insertion des jeunes. L'expérience des carrefours jeunesse-emploi ». Québec : Presses de l'Université du Québec. 
2010

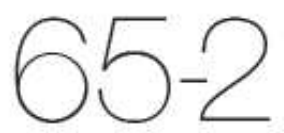

PRINTEMPS SPRING

REVUE TRIMESTRIELLE

RELATIONS INDUSTRIELLES

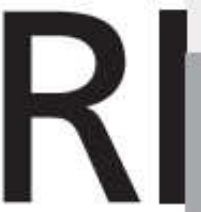

Revue trimestrielle bilingue publiée depuis 1945 par le

Departement des relations industrielles de l'Université Laval

\section{ARTICLES}

Penser le renouveau syndical par la sphère financière

URWANA COIQUAUD ET LUCIE MORISSETTE

Les logiques d'action collective d'associations regroupant des travailleurs indépendants MARTINE D'AMOURS

\section{RI/IR EN UGNE}

RI/IR est disponible en ligne sur le site Erudit: wnw.erudit.org/revue/ri

Pour abonnement à la version numérique, contacter Érudit.

Pour consulter les sommaires des numéros publiés, les résumés d'articles ou pour vous abonner à la version papier, visitez notre site Internet: www.riir.ulaval.ca

\section{INDUSTRIAL RELATIONS \\ QUARTERLY REVIEW}

A bilingual quarterly published since 1945 by the Industrial Relations Department, Universite Laval

\section{ARTICLES}

Employer Offered Family Support Programs and Voluntary and Involuntary Part-Time Work ISIK U. ZEYTINOGLU, GORDON B. COOKE AND SARA L. MANN Industrial Voluntarism in Canada SEAN TUCKER AND ALEX MUCALOV Toward a Career Anchor Structure: An Empiricial Investigation of Engineers LAURA WILS, THIERRY WILS AND MICHEL TREMBLAY Progress on Women's Equality within UK and Canadian Trade Unions: Do Women's Structures Make a Difference? JANE PARKER AND JANICE FOLEY

Effectiveness of Canada's Employment Equity Legislation for Women (1997-2004): Implications for Policy Makers

HARISH C. JAIN, JOHN I. LAWLER, BING BAI AND EUN KYUNG LEE

\section{RI/IR ON LINE}

RI/IR is available on line on Erudit website at: www.erudit.org/revue/ri

To subscribe to the online version, please contact Erudit.

Visit our website for contents listings, abstracts, or to subscribe to the print edition: www.riir.ulaval.ca

\section{RELATIONS INDUSTRIELLES INDUSTRLAL RELATIONS}

Pavillon J.-A.-DeSeve, 1025, avenue des Sciences-Humaines Bureau 3129, Université Laval, Québec (QC), Canada, G1V OA6 TELEPHONE : (418) 656-2468 TELECOPIEUR / FAX : (418) 656-3175 COURRIEL / E-MAIL : relat.inderit.ulaval.ca

wwww.riir.ulaval.ca 